\title{
Motor imagery performance and tactile acuity in patients with complaints of arms, neck and shoulder
}

Citation for published version (APA):

Heerkens, R. J., Koke, A. J. A., Lotters, F. J. B., \& Smeets, R. J. E. M. (2018). Motor imagery performance and tactile acuity in patients with complaints of arms, neck and shoulder. Pain Management, 8(4), 277286. https://doi.org/10.2217/pmt-2017-0070

Document status and date:

Published: 01/07/2018

DOI:

10.2217/pmt-2017-0070

Document Version:

Publisher's PDF, also known as Version of record

Document license:

Taverne

Please check the document version of this publication:

- A submitted manuscript is the version of the article upon submission and before peer-review. There can be important differences between the submitted version and the official published version of record.

People interested in the research are advised to contact the author for the final version of the publication, or visit the DOI to the publisher's website.

- The final author version and the galley proof are versions of the publication after peer review.

- The final published version features the final layout of the paper including the volume, issue and page numbers.

Link to publication

\footnotetext{
General rights rights.

- You may freely distribute the URL identifying the publication in the public portal. please follow below link for the End User Agreement:

www.umlib.nl/taverne-license

Take down policy

If you believe that this document breaches copyright please contact us at:

repository@maastrichtuniversity.nl

providing details and we will investigate your claim.
}

Copyright and moral rights for the publications made accessible in the public portal are retained by the authors and/or other copyright owners and it is a condition of accessing publications that users recognise and abide by the legal requirements associated with these

- Users may download and print one copy of any publication from the public portal for the purpose of private study or research.

- You may not further distribute the material or use it for any profit-making activity or commercial gain

If the publication is distributed under the terms of Article $25 \mathrm{fa}$ of the Dutch Copyright Act, indicated by the "Taverne" license above, 


\title{
Motor imagery performance and tactile acuity in patients with complaints of arms, neck and shoulder
}

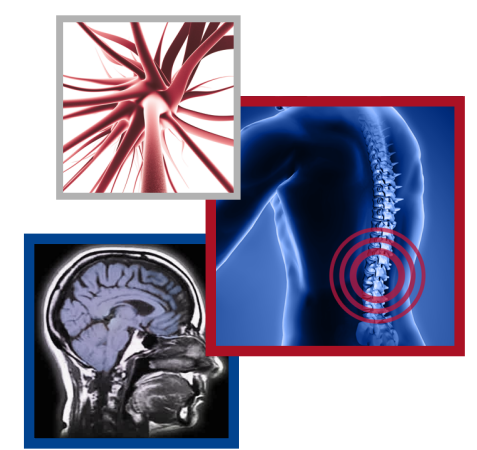

\author{
Renée J Heerkens ${ }^{1}$, Albère JA Köke*,2,3,4, Freek JB Lötters ${ }^{5}$ \& Rob JEM Smeets ${ }^{2,6}$ \\ ${ }^{1}$ Heliomare, Centre for Rehabilitation, Wijk aan Zee, The Netherlands \\ ${ }^{2}$ Maastricht University, Department of Rehabilitation Medicine, Maastricht, The Netherlands \\ ${ }^{3}$ Adelante Centre of Expertise in Rehabilitation, Hoensbroek, The Netherlands \\ ${ }^{4}$ South University of Applied Sciences, Department of Physiotherapy, Heerlen, The Netherlands \\ ${ }^{5}$ Hand \& Wrist Center, Dordrecht, The Netherlands \\ ${ }^{6}$ Libra Rehabilitation \& Audiology, Eindhoven/Weert, The Netherlands \\ *Author for correspondence: Tel.: +31 433882 162; albere.koke@maastrichtuniversity.nl
}

\section{Practice points}

- Accuracy and response time for motor imagery performance is not different between complaints of arm, neck and shoulder patients and patients without arm pain.

- Two-point discrimination measurement requires a standardized testing protocol.

- Both tactile acuity and motor imagery performance were not related to clinical factors like pain intensity and disability.

- Tactile acuity at the painful arm is worse than tactile acuity of the nonpainful arm in patients with CANS.

- In addition to earlier findings on two-point discrimination in other pain states, the decreased tactile acuity in complaints of arm, neck and shoulder patient suggests a disruption of sensory feedback in chronic pain in general.

Aim: This study aims to gain more knowledge of the sensorimotor incongruence in patients with chronic nonspecific complaints of arm, neck and shoulder. Method: Seven patients and seven healthy controls performed a left/right judgment task, and tactile acuity was assessed by the two-point discrimination threshold at fingers and shoulders. Results \& conclusion: The results suggest a decreased tactile acuity in patients with chronic nonspecific complaints of arm, neck and shoulder and a faster reaction time at the painful arm, which might imply disturbed information processing of sensory and motor feedback. Due to the small sample size and low scores on the pain and disability questionnaires, these conclusions should be interpreted with care. Further research is recommended.

First draft submitted: 8 December 2017; Accepted for publication: 26 April 2018; Published online:

1 June 2018

Keywords • CANS • chronic pain • complaints of arm, neck and shoulder • motor imagery • sensorimotor incongruence $\bullet$ tactile acuity

Complaints of arm, neck and shoulder (CANS) include several conditions in which pain of the upper limb is involved. CANS is defined as: "Complaints of arm, neck and shoulder are musculoskeletal complaints of arm, neck and shoulder which are not caused by acute trauma or by any systemic disease" [1]. Etiology of CANS includes several factors within the biopsychosocial model. Physical factors, such as repetitive strain, as well as the psychosocial factors like stress or fear of movement, contribute to the development of complaints [2]. Stress, depression, fear-avoidance, coping, pain-related fear and catastrophizing seem to counteract the recovery and are related to the persistence of symptoms. Psychosocial factors appear to contribute even more to pain disability than biomedical or biomechanical factors [3,4].

At this moment, complete reduction of pain and disability for CANS is hard to achieve, probably due to the multifactorial causality. Exercise therapy is likely the best treatment option, but strong evidence is lacking [5,6]. 
New insights in pain mechanism may be promising for developing more effective treatment options for CANS. First, neuroimaging studies in patients with chronic pain have shown altered cortical activation patterns. This so called cortical reorganization takes place in the primary somatosensory cortex that corresponds to the affected limb or area of the body [7-11]. For example, in the complex regional pain syndrome (CRPS) and chronic low back pain, this is caused by central sensitization and disinhibition of the surrounding neural network in the cortex, which results in shrinkage or shifting of the representation of that body part in the somatosensory cortex [7,8,10-14]. These cortical changes are followed by a decreased tactile acuity - for example, the ability to discriminate between two tactile stimuli $[15,16]$ and pain score [10]. The evidence that tactile acuity is altered in patients with several pain syndromes, like CRPS [9,10,15], osteoarthritis (OA) [17] and chronic low back pain [18-20], is growing (see Catley et al. [21] for a review).

Training the ability to discriminate between two tactile stimuli provides promising results in the treatment of CRPS [10,22,23]. It also leads to a regain of cortical map size in the somatosensory cortex and less disability [13,24]. Second, the sensorimotor incongruence - that is, the conflict between the expected input of bodily sensations and the real sensory feedback the body receives from movement, may play a role in pain. In order to perform a movement adequately, the brain primarily makes an estimation of its sensory results, which is evaluated when the movement is executed, based on sensory feedback via the cortical maps [24,25]. Changes in these cortical sensorimotor maps due to reorganization may provoke an incongruence between predicted and actual sensory feedback, corrupted motor output and lead to pain and other sensory disturbances (determine position of the limb, feelings of distorted shape) $[15,18]$.

So, the sensorimotor incongruence involves both movement intention and sensory feedback, and relies on the spatial awareness of the body - for example, the awareness of the body's position in space. This so called body schema is an online representation of one's peripersonal space which integrates different types of input, such as visual, proprioceptive and tactile input and interacts with motor systems - that is, in guiding of movements [22,26,27].

One way to evaluate the working body schema is by measuring the motor imagery performance (MIP) by performing a left/right judgment task in which pictures of, for instance, hands are displayed in several positions and one has to judge whether a left or a right hand is shown. The process of performing such a task involves three steps of implicit imagined movement. First, a spontaneous interpretation is made. Second, the person mentally maneuvers his own limb until the displayed position is imitated, and eventually this position is being verified. When the primarily response is wrong, the whole process starts again [28]. Several recent studies have shown an impaired MIP of the affected limb in different chronic pain states, like CRPS [29,30], carpal tunnel syndrome [31] and OA [32]. Training MIP is part of the graded motor imagery program that shows positive results regarding pain in patients with CRPS [33,34].

Whether MIP and tactile acuity are also disrupted in patients with CANS, and whether these disruptions are associated with higher pain and disability is unknown. Yet understanding whether and how these 'mechanisms' are involved, enables us to develop more effective treatment programs for these patients. Moreover, knowledge about MIP and TA in CANS patients might also lead to a better understanding of chronic pain in general.

We hypothesized that in patients with CANS, the motor imagery task was performed less accurate and slower at the hand and shoulder of the painful limb when compared with the hand and shoulder of the corresponding limb in healthy controls and to their own healthy side. For two-point discrimination (TPD), we hypothesized a lower accuracy at the painful side when compared with that side in healthy controls. We also hypothesized that in CANS patients, the TPD threshold is higher at the index finger, shoulder and most painful site at the painful arm compared with the nonpainful arm.

\section{Methods}

Participants

From October 2014 until November 2015, patients with nonspecific CANS were recruited from the department of rehabilitation of Maastricht University Medical Centre (MUMC) and the Handencentrum Utrecht, The Netherlands, for participation in this study. Patients with unilateral nonspecific CANS [1], symptom duration longer than 3 months, limitations in daily life functioning due to CANS and 18 years or older were included. Patients with specific causes (trauma, severe facet joint arthrosis of the cervical spine or systematic diseases - for example, rheumatoid arthritis or OA, Raynaud disease) of upper limb pain, neurological symptoms (limb weakness, sensory loss, isolated atrophy), frozen shoulder, local pain accompanied by swelling and redness, trigger finger with local pain and/or carpal tunnel syndrome were excluded. 


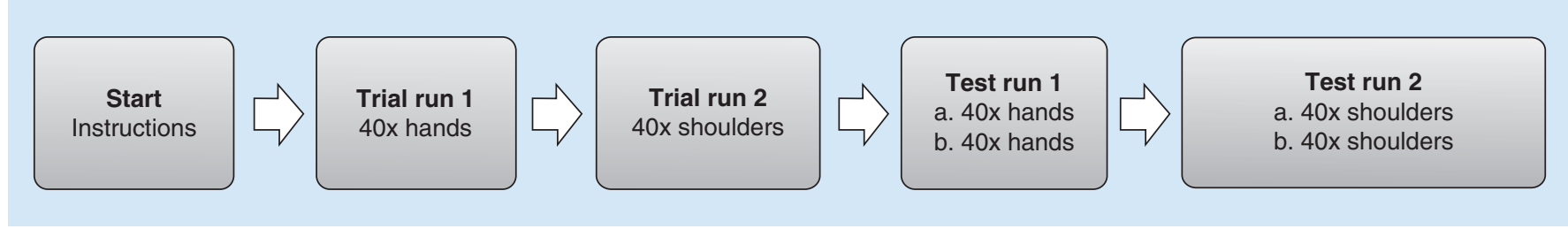

Figure 1. Overview of test order.

Healthy controls were recruited by an advertising poster at Maastricht University. Controls were included if no complaints of the upper limbs were present at the moment of assessment. Complaints in the past were documented.

The attending physiatrist recruited patients who were treated for CANS at the Handencentrum or at the department of rehabilitation medicine at MUMC. Patients who received treatment in the past 5 years at the latter department were invited for participation by letter. Patients who still suffered from CANS were asked to participate. The MUMC ethics committee approved the study (14-4-135). All participants gave their written consent to participate in this study.

\section{Measurements}

All measurements were performed during a single assessment at the MUMC or at the Handencentrum Utrecht.

\section{Questionnaires}

Participants filled in a questionnaire about sociodemographic information and their complaints, which included gender, age, height, weight, hand dominance and side, location and duration of complaints.

Current pain and average pain intensity during the last $48 \mathrm{~h}$ were measured by the visual analog scale (range 0-100 $\mathrm{mm}$ with $0=$ no pain and $100=$ worst pain imaginable) [35], which is a valid instrument for pain measurement [36]. Disabilities due to complaints of the upper limb were assessed by the Dutch Disabilities of the Arm, Shoulder and Hand questionnaire (DASH-DLV, 30 items with a score range 1-5 per item with $1=$ no difficulty and 5 = unable), a valid instrument for assessing disability of the upper limb $[37,38]$.

\section{Motor imagery performance}

To measure MIP, the Recognize ${ }^{T M}$ program (obtained online from www.noigroup.com/recognise) was used. In this computer program, the participant is asked to judge the laterality of the shown picture of a hand or shoulder, which are shown in different positions. The participant received instructions to respond as accurately and as quickly as possible, by pressing the corresponding arrow keys on the keyboard - for example, when he thought a right hand/shoulder was shown, he had to strike the right arrow key. Each picture was shown for $5 \mathrm{~s}$ or until the participant responded. If response extended $5 \mathrm{~s}$, the next picture was shown and the response was counted as a failure. Patients conducted two practice runs to make sure that the results could not be attributed to learning effects [39]. See Figure 1 for a flow diagram. The average scores of accuracy and recognition time of two runs were used as outcome measures. In between test runs there was a 2 min break [40]. Furthermore, participants were instructed not to move their upper limbs, to make sure the imagined movement was measured. The 'vanilla' setting for difficulty was used. Furthermore the recognition test for the neck was not used as they were not available and because only patients with unilateral complaints of the arm/shoulder were included.

\section{Two-point discrimination}

To measure tactile acuity, the TPD threshold was determined by using a mechanical caliper (baseline aesthesiometer) with a precision of $1 \mathrm{~mm}$ and a range from 2 to $103 \mathrm{~mm}$. TPD measurement was performed following the procedure (staircase method) described by Moberg [41]. As pain was nonspecific, not restricted to a specific (dermatomal) distribution, the TPD in the painful region was measured in a transverse direction, (adapted to the pain area) at both arms at the lateral shoulder near the glenohumeral joint (mid axillary line) and at the palmar side of the end phalanx of the index finger.

In patients, TPD was also measured at the most painful region and the same region at the nonpainful arm. To identify this region, patients were asked to point out the most painful area. 
At each site, measuring started with one point (e.g., $0 \mathrm{~mm}$ distance). The distance between the two points gradually increased until the participant felt two different points. From this point on, five ascending and five descending runs were executed with steps of $1 \mathrm{~mm}$ increase and decrease respectively, to determine the exact TPD threshold. The participant was instructed to say 'one' if he felt one point, 'two' if he felt two points and 'not sure' (forced choice method) if he was in doubt. Seven correct answers out of ten were accepted as valid for determination of the threshold [41]. The caliper was gently applied with little pressure until surrounding skin started to blanch [41]. Interstimulus interval was a minimum of $5 \mathrm{~s}$. During the test, the participant was instructed to close his eyes and not move his limbs [42]. TPD thresholds of the index fingers were measured first, followed by the shoulders and the most painful region. To avoid interobserver bias, all measurements were performed by one researcher. The researcher was blinded by asking the patient not to reveal the side and site of complaints until the most painful site had to be tested.

\section{Statistical analysis}

SPSS 23.0 was used for statistical analysis. Because of the small sample size, nonparametric statistics were used. The demographic characteristics of participants were calculated as medians and quartiles.

To detect differences in the MIP task and tactile acuity between patients and controls, MIP scores and TPD thresholds were compared between patients and controls. As all patients reported pain in the right arm, the results of the right arm (i.e., hand and shoulder for MIP, and finger index/shoulder for TPD threshold) of this group were compared with the results of the right arm of the controls, respectively. Likewise, the results of the left arm were compared between both groups. For MIP, both accuracy (percentage of correct answers) and speed data (recognition time) for the hand and shoulder were used for analysis. To detect these between group differences Mann-Whitney $\mathrm{U}$ tests were used.

To detect differences between the painful and nonpainful arm, data of the patients were used only. For TPD, results of the shoulder, index finger and most painful region according to the patient were compared between painful and nonpainful side in CANS patients. For MIP, results of the shoulder and hand were compared likewise. To detect these within-group differences, Wilcoxon signed-rank tests were performed.

Correlations between both TPD and MIP (accuracy and reaction time) of the affected shoulder and index finger/hand, pain intensity (both current pain and average pain scores) and disability were calculated for the CANS group with Spearman's correlation coefficients.

For calculating the influence of age on tactile acuity, Spearman's correlation coefficients with data of the rightsided index finger of healthy subjects were used, after controlling for right- and left-sided differences by Wilcoxon signed-rank test.

\section{Results}

A total of 49 patients were approached for participation, of which 10 were screened for participation. Of these patients, three were recruited by letter and seven by admission through the out-patient clinic. Two patients were excluded during the screening; one person had complaints since only 1 month and the other was diagnosed with Lyme's disease. So in total, eight patients with nonspecific CANS were measured. However, as one patient seemed to have complaints at both arms during measurement, meaning there was no 'healthy' side, this patient was also excluded. The control group consisted of seven participants. All the patients had right-sided complaints, so all the data concerning the affected limb could be compared with the data from the right arms from the healthy participants. All participants in the control group were right handed. In the patient group, six patients were right handed and one patient was left handed.

Demographic data of the included participants are summarized in Table 1. Patients were statistically significant older than controls.

\section{Motor imagery performance}

There was no difference in accuracy between people with CANS and the control group at the affected arm (hand: $[Z=-1,132 ; p=0.258]$, shoulder: $[Z=-0.860 ; p=0.390]$ ) and the nonaffected arm (hand $[Z=-0.259 ; p=0.795]$, shoulder $[Z=-1.1510 ; p=0.131])$; neither did recognition time differ between patients with CANS and healthy controls, measured at the affected arm (hand $[Z=-0.128 ; p=0.898]$, shoulder $[Z=-0.384 ; p=0.701]$ ) and nonaffected arm (hand $[Z=0.000 ; p=1.00]$, shoulder $[Z=-0.385 ; p=0.701]$ ). Table 2 within-patient analysis showed no differences in accuracy between the painful and nonpainful arm, but a significant difference was found 
Table 1. Participant demographics.

\begin{tabular}{|c|c|c|c|}
\hline Variables & $\begin{array}{l}\text { CANS } \\
(n=7)\end{array}$ & $\begin{array}{l}\text { Controls } \\
(n=7)\end{array}$ & Significance \\
\hline Age (years) & $43.0(39.0-50.0)$ & $26.0(24.0-27.0)$ & $p=0.017$ \\
\hline $\begin{array}{l}\text { Gender }(n): \\
\text { - Male } \\
\text { - Female }\end{array}$ & $\begin{array}{l}3 \\
4\end{array}$ & $\begin{array}{l}1 \\
6\end{array}$ & \\
\hline $\begin{array}{l}\text { Hand dominance }(n) \text { : } \\
\text { - Left hand } \\
\text { - Right hand }\end{array}$ & $\begin{array}{l}1 \\
6\end{array}$ & $\begin{array}{l}0 \\
7\end{array}$ & \\
\hline $\begin{array}{l}\text { Most painful region }(n) \text { : } \\
\text { - Right } \\
\text { - Hand } \\
\text { - Shoulder } \\
\text { - Neck } \\
\text { - Lower arm }\end{array}$ & $\begin{array}{l}9^{\dagger} \\
3 \\
4 \\
1 \\
1\end{array}$ & NA & \\
\hline Duration of pain episode (years) & $5.0(4.0-15.0)$ & NA & \\
\hline $\begin{array}{l}\text { Pain intensity } \ddagger \\
\text { - Current } \\
\text { - Average (<48 h) }\end{array}$ & $\begin{array}{l}16.0(6.0-35.0) \\
29.0(20.0-61.8)\end{array}$ & NA & \\
\hline Disability ${ }^{\S}$ & $12.5(6.7-40.2)$ & NA & \\
\hline \multicolumn{4}{|c|}{$\begin{array}{l}\text { All values are median (quartiles). } \\
\text { †Two patients reported more than one region as the most painful. } \\
\text { ¡Pain intensity measured on a } 0-100 \mathrm{~mm} \text { visual analog scale. } \\
\text { § Disability measured by Disabilities of Arm, Shoulder and Hand questionnaire. } \\
\text { CANS: Complaints of arm, neck and shoulder; E: Elbow; H: Hand; L: Lower arm; n: Number of participant; N: Neck; NA: Not applicable; S: Shoulder; W: Wrist. }\end{array}$} \\
\hline
\end{tabular}

\begin{tabular}{|c|c|c|c|c|}
\hline Variables & CANS affected side & $\begin{array}{l}\text { Control group matched to } \\
\text { affected side }\end{array}$ & CANS unaffected side & $\begin{array}{l}\text { Control group matched to } \\
\text { unaffected side }\end{array}$ \\
\hline \multicolumn{5}{|c|}{ Recognition accuracy in \% } \\
\hline Hands & $95.00(87.50-95.00)$ & $87.50(75.00-95.00)$ & $95.00(77.50-100.00)$ & $95.00(80.00-97.50)$ \\
\hline Shoulders & $97.50(92.50-100.00)$ & $97.50(92.50-97.50)$ & $100.00(97.50-100.00)$ & $95.00(95.00-100.00)$ \\
\hline \multicolumn{5}{|c|}{ Recognition time in seconds } \\
\hline Hands & $1.75(1.45-42.45)$ & $1.90(1.25-22.20)$ & $1.75(1.40-42.50)$ & $2.05(1.30-32.35)$ \\
\hline Shoulders & $1.40(1.30-31.60)$ & $1.25(1.10-11.75)$ & $1.50(1.35-31.75)$ & $1.50(1.15-11.75)$ \\
\hline \multicolumn{5}{|c|}{ TPD threshold in $\mathrm{mm}$} \\
\hline Index finger & $4.00(4.00-04.00)$ & $3.00(2.00-03.00)$ & $3.00(3.00-04.00)$ & $2.00(2.00-03.00)$ \\
\hline Shoulder & $48.00(46.00-56.40)$ & $33.00(30.00-37.00)$ & $42.00(39.00-56.40)$ & $35.00(29.00-35.00)$ \\
\hline
\end{tabular}

in recognition time, whereas pictures of shoulders of the painful side were recognized significantly faster $(\mathrm{Z}=$ -2.032; $\mathrm{p}=0.042)$.

MIP versus pain

No significant correlation between accuracy or recognition time of the shoulder, hand and current pain and average pain were found (Table 3). 
Table 3. Spearman's rho correlation coefficients for motor imagery performance (accuracy and reaction time) versus current pain, average pain and disability.

\begin{tabular}{|c|c|c|c|c|}
\hline \multirow[t]{2}{*}{ Variables } & \multicolumn{2}{|c|}{ Accuracy } & \multicolumn{2}{|c|}{ Recognition time } \\
\hline & Hand & Shoulder & Hand & Shoulder \\
\hline Current pain & $-0.259(0.574)$ & $-0.599(0.155)$ & $0.643(0.119)$ & $0.198(0.670)$ \\
\hline Average pain $(<48 \mathrm{~h})$ & $0.259(0.574)$ & $-0.299(0.514)$ & $0.036(0.939)$ & $0.144(0.758)$ \\
\hline Disability & $-0.259(0.574)$ & $-0.898(0.006)$ & $0.571(0.180)$ & $0.613(0.144)$ \\
\hline
\end{tabular}

MIP versus disability

At the shoulder, but not for the hand, a significant negative correlation was found between MIP and disability. This means that a higher disability was related to lower accuracy. The correlation between recognition time and disability was not significant for both hand and shoulder (Table 3).

\section{Tactile acuity}

A significant difference between TPD in CANS patients and the control group at both regions, measured at the affected side, was found. CANS patients had higher TPD thresholds (index finger: $Z=-2.539 ; \mathrm{p}=0.010$, shoulder: $\mathrm{Z}=3.023 ; \mathrm{p}=0.003$ ). At the unaffected side, TPD thresholds in CANS patients were also significantly higher at both regions (index finger: $Z=-2.225 ; p=0.038$, shoulder: $Z=-3.148 ; p=0.001$ ) (Table 2).

Within-patient analysis showed higher TPD thresholds at the painful arm, with a statistically higher TPD at the index finger $(\mathrm{Z}=-2.121 ; \mathrm{p}=0.034)$. TPD threshold at the shoulder was also higher, but not statistically significant $(\mathrm{Z}=-1.625 ; \mathrm{p}=0.104)$.

A total of nine regions were pointed out as being the most painful regions (Table 1), meaning that some patients pointed out more than one region as being the most painful. A significantly higher TPD threshold was found for the painful regions at the painful arm $(Z=-2.035 ; p=0.042)$ when compared with the same regions of the nonpainful arm.

\section{TPD versus pain}

For both current and average pain, no significant correlations with TPD threshold were found at the index finger and shoulder (current $[\rho=-0.267 ; \mathrm{p}=0.57],<48 \mathrm{~h}[\rho=0.267 ; \mathrm{p}=0.57]$ ) and shoulder (current $[\rho=0.000$; $\mathrm{p}=1.000],<48 \mathrm{~h}[\rho=0.418 ; \mathrm{p}=0.35])$.

\section{TPD versus disability}

No significant correlation was found between disability and TPD threshold at the index finger $(\rho=0.267 ; \mathrm{p}=0.57)$ nor shoulder $(\rho=0.109 ; \mathrm{p}=0.82)$.

\section{Discussion}

This was the first time MIP and tactile acuity were measured in CANS patients.

Outcomes of MIP were divided into accuracy and recognition time, both reflecting a different process of the task, with accuracy reflecting the working body schema and recognition time reflecting the information processing or initial estimation. In this study, accuracy did not differ between patients with nonspecific CANS and healthy controls. Also, accuracy was equal between the painful and nonpainful arm in CANS patients. This is in contrast to previous studies on MIP in other chronic pain conditions, in which a decreased accuracy was found at the painful region [29,31-33].

These conflicting results might be due to differences in the test protocols used (trial run, amount of pictures used and difficulty of pictures) to measure MIP (i.e., accuracy). In the present study, measurement was preceded by a trial run of 80 pictures to exclude a learning effect [39]. Other studies did not implement a trial run or used less pictures in the trial run, which means the learning effect might have been present during testing. Also the amount of pictures used differed in all previous studies and was lower than in the present study. This again might have resulted in a learning effect, leading to the potentially incorrect conclusion that patients have a lower accuracy at their affected limb. 
Another difference in the test protocol might be the level of difficulty. However, the pictures used in other studies seem quite similar to the pictures used by the Recognize when for instance rotation angles are compared. Stanton et al. [32] used the exact same difficulty level of the Recognize program and did find an impaired accuracy in patients with knee OA and patients with upper limb pain. However, they did not control for possible learning effects.

Also no difference in recognition time was found between CANS patients and the controls, however within patient analysis showed that pictures of shoulders of the painful limb were recognized faster than pictures of the nonpainful shoulder. This finding is not congruent with one of our hypotheses and might reflect a disruption of information processing with a bias toward the painful side as described by Hudson et al. [43]. They hypothesized that due to this bias to the affected side, a person interprets a picture of the unaffected side wrong, which needs to be corrected and therefore takes more time. To confirm this information biasing, one would expect to find this slower reaction time for pictures showing shoulders as well as hands of the unaffected limb. Additionally, no significant correlation was found with recognition time and level of pain intensity, which is contrary to this theory of biased information processing. However, this nonsignificant correlation might also be attributed to the relative low number of participants with CANS.

Finding no significant correlation between pain intensity and MIP (accuracy and recognition time) might also be due to the severity of complaints of our patient group. Most patients were recruited during or after treatment and had relatively low pain scores. Only three patients reported moderate pain during the last $48 \mathrm{~h} \mathrm{(>49} \mathrm{mm} \mathrm{on}$ visual analog scale [36]) and only one patient reported moderate current pain. However, we did find that a lower accuracy at the shoulder was significantly correlated with higher disability scores. This was not found for the hand. A plausible explanation for this finding would be highly suggestive, given the rather low number of participants, and more research to replicate or refute this finding is necessary.

Tactile acuity appeared to be decreased in patients with CANS. In fact, TPD thresholds were significantly larger in patients with CANS when compared with controls for all tested areas of both the painful and nonpainful arm. Furthermore, tactile acuity at the most painful region as well as in other regions of the painful arm was worse compared with the nonpainful arm in CANS patients, suggesting a disrupted information processing at the painful limb. This is the first time these findings are described for CANS, and they confirm earlier literature about disturbed tactile acuity in other chronic pain states [9,10,15,17-21].

In this study, correlations between pain and tactile acuity were all nonsignificant, but this can be due to the small study sample and rather low pain severity. So whether, and how pain and tactile acuity are related in CANS patients remains unclear.

Our patients rated pain intensity probably on the basis of the most painful site, which in several patients did not reflect the pain experienced at the hand or shoulder, where TPD was measured. Yet for analyzing the correlation between pain intensity and TPD threshold, the TPD thresholds of the shoulder and index finger of all patients were used. It would definitely be useful to calculate this correlation between TPD at the painful site, however as all regions have different TPD values due to a proximal-distal gradient in receptive fields [44], but our sample was far too small to do this additional analysis.

TPD and disability were not significantly correlated. This might be explained by the relatively low disability scores, with only two patients scoring higher than the normative value on the DASH [45] (http://www.dash.iwh.on.ca/). Also we only used self-reported disability, which might not reflect real daily activity levels.

It is known that tactile acuity decreases with age [46]. Our control group was significantly younger. However post hoc analysis, using Spearman rho's correlation coefficients, showed that there was no influence of age on MIP and TPD.

\section{Study limitations \& implications for further research}

The main limitation of this study is the small sample size. While inclusion of patients went laboriously, controls were already recruited and thereby matching to age and gender was not possible. Additionally, included patients had no severe complaints. Probably because they were recruited during or after treatment, resulting in rather low pain intensity and disability scores. The results presented in this report should therefore be interpreted with care. We also compared only the data form the right arm, which was the dominant arm for all controls and patients (except for one). We tested whether there was a difference for TPD between left and right side, however Wilcoxon signed-rank tests showed no significant difference $(\mathrm{p}=0.1)$. Therefore we assume no different findings when comparing the left arm and hand. 
Although a standard assessment protocol was used to perform the TPD measurement correctly, it appeared to be difficult to apply both stimuli at exactly the same time and with equal pressure at repetition, which was also affected by body shape or contour of shoulder or arm. Differences or asymmetry in body regions influence TPD measures [47]. As no data were collected on anthropometric characteristics, we could not rule out any bias of TPD score by differences in body shape or contour. Additionally, the chosen method to measure TPD appears to be a difficult task for patients. The assessment was time-consuming and demanded continuous concentration. People might tend to be confused because of uncertainty of the repeated stimuli and ratings (one, two or ?). So it is not unlikely that patients got tired or distracted. The importance of factors such as fatigue, cooperation, concentration and force stimuli is documented [48-50]. To minimize the chance of tiring or distraction, patients were offered a short break after completing a skin region. However both factors could have influenced the results.

\section{Conclusion}

The aim of this present study was to gain more knowledge about the sensorimotor incongruence by measuring MIP and tactile acuity in patients with CANS. For MIP (accuracy and recognition time), no differences were found between patients and controls. In CANS patients, the recognition time of shoulder pictures was shorter at the painful side, implying an information processing bias toward the painful side. However, this was not found for the hand. Tactile acuity was decreased in patients with CANS at the hand and shoulder of the painful and nonpainful arm, with tactile acuity being worse at the painful arm, suggesting that in CANS patient, the spatially sensory feedback is disrupted. However, these findings did not significantly correlate with pain intensity and disability, so it is unclear what this finding means for clinical application/reasoning.

Because of the study limitations, no firm conclusions can be drawn. Further research, with a bigger sample, is therefore needed. To ensure sufficient reliability in future research, we highly recommend standardization of the protocol and training.

Acknowledgements

The authors would like to thank the patients and volunteers for participation in this study.

Financial \& competing interests disclosure

The authors have no relevant affiliations or financial involvement with any organization or entity with a financial interest in or financial conflict with the subject matter or materials discussed in the manuscript. This includes employment, consultancies, honoraria, stock ownership or options, expert testimony, grants or patents received or pending, or royalties.

No writing assistance was utilized in the production of this manuscript.

Ethical conduct of research

The MUMC ethics committee approved the study (14-4-135). All participants gave their written consent.

\section{References}

1. Huisstede BM, Miedema HS, Verhagen AP, Koes BW, Verhaar JA. Multidisciplinary consensus on the terminology and classification of complaints of the arm, neck and/or shoulder. Occup. Environ. Med. 64(5), 313-319 (2007).

2. Van Eijsden-Besseling MD, van Attekum A, de Bie RA, Staal JB. Pain catastrophizing and lower physical fitness in a sample of computer screen workers with early non-specific upper limb disorders: a case-control study. Ind. Health 48(6), 818-823 (2010).

3. Linton SJ. A review of psychological risk factors in back and neck pain. Spine 25(9), 1148-1156 (2000).

4. Pincus T, Burton AK, Vogel S, Field AP. A systematic review of psychological factors as predictors of chronicity/disability in prospective cohorts of low back pain. Spine 27(5), E109-E120 (2002).

5. Verhagen AP, Karels C, Bierma-Zeinstra SM et al. Exercise proves effective in a systematic review of work-related complaints of the arm, neck, or shoulder. J. Clin. Epidemiol. 60(2), 110-117 (2007).

6. Verhagen AP, Bierma-Zeinstra SM, Burdorf A et al. Conservative interventions for treating work-related complaints of the arm, neck or shoulder in adults. Cochrane Database Syst. Rev. 19(3), CD008742 (2013).

7. Maihofner C, Handwerker HO, Neundorfer B, Birklein F. Patterns of cortical reorganization in complex regional pain syndrome. Neurology 61(12), 1707-1715 (2003).

8. Juottonen $\mathrm{K}$, Gockel M, Silén T et al. Altered central sensorimotor processing in patients with complex regional pain syndrome. Pain 98(3), 315-323 (2002).

9. Pleger B, Tegenthoff M, Schwenkreis $\mathrm{P}$ et al. Mean sustained pain levels are linked to hemispherical side-to-side differences of primary somatosensory cortex in the complex regional pain syndrome I. Exp. Brain Res. 155(1), 115-119 (2004). 
10. Pleger B, Tegenthoff M, Ragert $\mathrm{P}$ et al. Sensorimotor retuning [corrected] in complex regional pain syndrome parallels pain reduction. Ann. Neurol. 57(3), 425-429 (2005).

11. Flor $\mathrm{H}$, Braun C, Elbert T, Birbaumer N. Extensive reorganization of primary somatosensory cortex in chronic back pain patients. Neurosci. Lett. 224(1), 5-8 (1997).

12. McCabe CS, Haigh RC, Halligan PW, Blake DR. Referred sensations in patients with complex regional pain syndrome type 1 . Rheumatology 42(9), 1067-1073 (2003).

13. Maihöfner C, Handwerker HO, Neundörfer B, Birklein F. Cortical reorganization during recovery from complex regional pain syndrome. Neurology 63(4), 693-701 (2004).

14. Pleger B, Ragert P, Schwenkreis $\mathrm{P}$ et al. Patterns of cortical reorganization parallel impaired tactile discrimination and pain intensity in complex regional pain syndrome. Neuroimage 32(2), 503-510 (2006).

15. Lotze M, Moseley GL. Role of distorted body image in pain. Curr. Rheumatol. Rep. 9(6), 488-496 (2007).

16. Gallace A, Spence C. Touch and the body. The role of the somatosensory cortex in tactile awareness. Psyche 16, 30-67 (2006).

17. Stanton TR, Lin CW, Bray H et al. Tactile acuity is disrupted in osteoarthritis but is unrelated to disruptions in motor imagery performance. Rheumatology 52(8), 1509-1519 (2013).

18. Moseley GL. I can't find it! Distorted body image and tactile dysfunction in patients with chronic back pain. Pain 140(1), 239-243 (2008).

19. Luomajoki H, Moseley GL. Tactile acuity and lumbopelvic motor control in patients with back pain and healthy controls. Br. J. Sports Med. 45(5), 437-440 (2011).

20. Nishigami T, Mibu A, Osumi M et al. Are tactile acuity and clinical symptoms related to differences in perceived body image in patients with chronic nonspecific lower back pain? Man. Ther. 20(1), 63-67 (2015).

21. Catley MJ, O'Connell NE, Berryman C, Ayhan FF, Moseley GL. Is tactile acuity altered in people with chronic pain? A systematic review and meta-analysis. J. Pain 15(10), 985-1000 (2014).

22. Moseley GL, Zalucki NM, Wiech K. Tactile discrimination, but not tactile stimulation alone, reduces chronic limb pain. Pain 137(3), 600-608 (2008).

23. Moseley GL, Flor H. Targeting cortical representations in the treatment of chronic pain: a review. Neurorehabil. Neural Repair 26(6), 646-652 (2012).

24. Bailey J, Nelson S, Lewis J, McCabe CS. Imaging and clinical evidence of sensorimotor problems in CRPS: utilizing novel treatment approaches. J. Neuroimmune Pharmacol. 8(3), 564-575 (2013).

25. Haggard P, Wolpert D. Higher-order motor disorders: from neuroanatomy and neurobiology to clinical neurology. In: Disorders Of Body Schema. Leiguarda R, Freud H, Jeannerod M, Hallet M (Eds). Oxford University Press, Oxford, UK (2005).

26. Gallace A1, Spence C. The cognitive and neural correlates of 'tactile consciousness': a multisensory perspective. Conscious Cogn. 17(1), 370-407 (2008).

27. Moseley GL, Gallace A, Spence C. Bodily illusions in health and disease: physiological and clinical perspectives and the concept of a cortical 'body matrix'. Neurosci. Biobehav. Rev. 36(1), 34-46 (2012).

28. Parsons LM. Integrating cognitive psychology, neurology and neuroimaging. Acta Psychol. 107(1-3), 155-181 (2001).

29. Schwoebel J, Friedman R, Duda N, Coslett HB. Pain and the body schema: evidence for peripheral effects on mental representations of movement. Brain 124(Pt 10), 2098-2104 (2001).

30. Moseley GL. Why do people with complex regional pain syndrome take longer to recognize their affected hand? Neurology 62(12), 2182-2186 (2004).

31. Schmid AB, Coppieters MW. Left/right judgment of body parts is selectively impaired in patients with unilateral carpal tunnel syndrome. Clin. J. Pain 28(7), 615-622 (2012).

32. Stanton TR, Lin CW, Smeets RJ, Taylor D, Law R, Lorimer Moseley G. Spatially defined disruption of motor imagery performance in people with osteoarthritis. Rheumatology 51(8), 1455-1464 (2012).

33. Bowering KJ, O'Connell NE, Tabor A et al. The effects of graded motor imagery and its components on chronic pain: a systematic review and meta-analysis. J. Pain 14(1), 3-13 (2013).

34. Moseley GL. Graded motor imagery is effective for long-standing complex regional pain syndrome: a randomised controlled trial. Pain 108(1-2), 192-198 (2004).

35. Collins SL, Moore RA, McQuay HJ. The visual analogue pain intensity scale: what is moderate pain in millimetres? Pain 72(1-2), 95-97 (1997).

36. McCormack HM, Horne DJ, Sheather S. Clinical applications of visual analogue scales: a critical review. Psychol. Med. 18(4), 1007-1019 (1988).

37. Hudak PL, Amadio PC, Bombardier C. Bombardier, development of an upper extremity outcome measure: the DASH (disabilities of the arm, shoulder and hand) [corrected]. The Upper Extremity Collaborative Group (UECG). Am. J. Ind. Med. 29(6), 602-608 (1996). 
38. Veehof MM, Sleegers EJ, van Veldhoven NH, Schuurman AH, van Meeteren NL. Psychometric qualities of the Dutch language version of the Disabilities of the Arm, Shoulder, and Hand questionnaire (DASH-DLV). J. Hand Ther. 15(4), 347-354 (2002).

39. Boonstra AM, de Vries SJ, Veenstra E, Tepper M, Feenstra W, Otten E. Using the Hand Laterality Judgement Task to assess motor imagery: a study of practice effects in repeated measurements. Int. J. Rehabil. Res. 35(3), 278-280 (2012).

40. Dey A, Barnsley N, Mohan R, McCormick M, McAuley JH, Moseley GL. Are children who play a sport or a musical instrument better at motor imagery than children who do not? Br. J. Sports Med. 46(13), 923-926 (2012).

41. Moberg E. Two-point discrimination test. A valuable part of hand surgical rehabilitation, e.g. in tetraplegia. Scand. J. Rehabil. Med. 22(3), 127-134 (1990).

42. Schaefer M, Heinze JH, Rotte M. Viewing touch improves tactile sensory threshold. Neuroreport 16(4), 367-370 (2005).

43. Hudson ML, McCormick K, Zalucki N, Moseley GL. Expectation of pain replicates the effect of pain in a hand laterality recognition task: bias in information processing toward the painful side? Eur. J. Pain 10(3), 219-224 (2006).

44. Weissman-Fogel I, Brayer-Zwi N, Defrin R. Spatial resolution of the pain system: a proximal-to-distal gradient of sensitivity revealed with psychophysical testing. Exp. Brain Res. 216(2), 181-190 (2012).

45. InstituteforWork \& Health The DASH measure. https://www.dash.iwh.on.ca/.

46. Kaneko A, Asai N, Kanda T. The influence of age on pressure perception of static and moving two-point discrimination in normal subjects. J. Hand Ther. 18(4), 421-424 (2005).

47. Falling C, Mani R. Regional asymmetry, obesity and gender determines tactile acuity of the knee regions: a cross-sectional study. Man. Ther. 26, 150-157 (2016).

48. Nolan MF. Two-point discrimination assessment in the upper limb in young adult men and women. Phys. Ther. 62(7), 965-969 (1982).

49. Lundborg G, Rosén B. The two-point discrimination test - time for a re-appraisal? J. Hand Surg. Br. 29(5), 418-422 (2004).

50. Catley MJ, Tabor A, Wand BM, Moseley GL. Assessing tactile acuity in rheumatology and musculoskeletal medicine - how reliable are two-point discrimination tests at the neck, hand, back and foot? Rheumatology 52(8), 1454-1461 (2013). 